\title{
An event-driven simulation for highest urgency first (HUF) : a latency and modulation aware bandwidth allocation algorithm for WiMAX base stations.
}

\begin{abstract}
Awide-spectrum of WiMAX simulators have been developed to provide analysis for advanced WiMAX system development, including the open source tool ns-2, and commercial software such as QualNet and OPNET Modeler. These tools are among the pertinent simulators, however,there are trade-offs such as complexity,large integrated components and licensing cost for commercial simulators. To strike an ideal balance in gauging these tradeoffs and to further complement the repository of simulators, this paper presents the details of a general-purpose programming language based discrete event simulation for WiMAX. This research has focused at developing a discrete-event simulator to implement bandwidth allocation algorithms for a WiMAX base station. The highest urgency first algorithm has been utilized as a benchmark deployment algorithm in order to provide: dynamic downlink/uplink adjustment and latency guarantee for real-time applications. Two performance analyses have been deployed for the purpose of extensive testing of the developed simulator. The analysis encompasses the modulation and coding scheme awareness and the latency guarantee with multiple and distinct requirements. The results show that the developed simulator has successfully produced the benchmark results. These findings have proved that the new simulator has successfully become a reliable option for WiMAX resource management performance analysis.
\end{abstract}

Keyword: WiMAX; Discrete event simulation; Scheduling algorithm; Simulation; Bandwidth allocation. 